\title{
NUMERICAL SIMULATION OF THE INTERACTION OF A REGULAR WAVE AND A SUBMERGED CYLINDER
}

\author{
P. R. F. Teixeira \\ Universidade Federal do Rio Grande \\ Escola de Engenharia \\ Avenida Itália, km8, Campus Carreiros \\ 96201-900, Rio Grande, RS, Brasil \\ pauloteixeira@furg.br
}

\begin{abstract}
A numerical simulation of the interaction between a regular wave and an immersed horizontal cylinder, whose axis is 3-radius deep, perpendicular to the direction of the wave propagation, is presented in this paper. The numerical model uses the semi-implicit two-step Taylor- Galerkin method to integrate Navier-Stokes equations in time and space. Arbitrary lagrangean-eulerian formulation is employed to describe the free surface movement. The free surface elevations near the cylinder and in some gauges along the channel, as well the spectrum distribution, are compared with experimental ones, and good agreement is obtained. The analysis shows that the viscous effects only affect the area that is very close to the cylinder.
\end{abstract}

Keywords: numerical simulations, finite element method, free surface, wave propagation, submerged cylinder

\section{NOMENCLATURE}

a wave amplitude, $\mathrm{m}$

$a_{i j} \quad$ influence coefficients, $\mathrm{m}^{-4}$

$c \quad$ sound speed, $\mathrm{m} / \mathrm{s}$

$d_{i j} \quad$ distance between points $i$ and $j, \mathrm{~m}$

$f \quad$ frequency wave, $\mathrm{Hz}$

$g_{i} \quad$ gravity acceleration components, $\mathrm{m} / \mathrm{s}^{2}$

$h_{\mathrm{E}} \quad$ characteristic element size, $\mathrm{m}$

$L \quad$ wavelength, $\mathrm{m}$

N linear shape function

$p$ pressure, $\mathrm{Pa}$

PE constant shape function

$\mathrm{r}$ cylinder ray, $\mathrm{m}$

$t$ time, $\mathrm{s}$

$T$ period, $\mathrm{s}$

$\mathrm{u}$ horizontal velocity component, $\mathrm{m} / \mathrm{s}$

$\mathrm{v} \quad$ vertical velocity component, $\mathrm{m} / \mathrm{s}$

$\mathrm{v}_{i} \quad$ fluid velocity components, $\mathrm{m} / \mathrm{s}$

$\mathrm{w}_{i} \quad$ mesh velocity components, $\mathrm{m} / \mathrm{s}$

$x c$ horizontal coordinate of the cylinder center, $\mathrm{m}$

$x_{i} \quad$ coordinates, $\mathrm{m}$

\section{Greek symbols}

$\begin{array}{ll}\beta & \text { safety factor } \\ \Delta t & \text { time step, } \mathrm{s} \\ \eta & \text { free surface elevation, } \mathrm{m} \\ \rho & \text { density, } \mathrm{kg} / \mathrm{m}^{3} \\ \tau_{i j} & \text { viscous stress tensor, } \mathrm{Pa}\end{array}$

\section{Superscripts}

$n \quad$ instant $t, \mathrm{~s}$

$n+1 / 2$ instant $t+\Delta t / 2, \mathrm{~s}$

$n+1 \quad$ instant $t+\Delta t, \mathrm{~s}$

\section{INTRODUCTION}

The interaction among regular waves and submerged circular cylinders, with their axes parallel to the crests of the incident waves, has been studied analytically, experimentally and numerically by many authors. The presence of an obstacle near the free surface may cause reflected and modified transmitted waves. These phenomena depend on the characteristics of the incident wave, the obstacle geometry and the depth. Many studies of this interaction are available to provide a good example to validate numerical codes.

The first study was developed by Dean (1948) and, after that, by Ursell (1950). Considering a linear behavior, these authors showed that (a) the cylinder does not reflect any energy, regardless of its ray, depth or wave frequency; (b) the transmitted waves are out of phase, but their amplitudes are the same. Chaplin (2001) studied the nonlinear forces and characteristics of the reflected and transmitted waves experimentally. He showed that the reflection is negligible up to the third order. This author and Schonberg and Chaplin (2003) presented many experimental and numerical studies concerning the nonlinear interaction among waves and submerged cylinders. A detailed review of analyses for this case can be found in Paixão Conde et al. (2007).

The objective of this paper is to validate the code developed by Teixeira (2001) in the interaction between a submerged body and a wave in a horizontal channel. Numerical results are compared with experimental ones in a wave over a submerged cylinder.

The model includes all nonlinear terms, different from the depth-integrated models, limited to smooth nonlinearities, although their computational cost is low. Therefore, the wave transformation due 
to a submerged obstacle can be accurately simulated in domains with relative little dimensions.

The code (Teixeira, 2001) integrates the full Navier-Stokes equations. A semi-implicit two-step Taylor-Galerkin method is used to simulate 3D incompressible flows with free surface. An arbitrary lagrangean-eulerian formulation (ALE) is used to enable relative movements among bodies and surfaces and the free surface movements.

\section{NUMERICAL MODEL}

\section{Semi-implicit two-step Taylor-Galerkin method}

Basically, the algorithm consists of the following steps (Teixeira and Awruch, 2000):

(a) Calculate non-corrected velocity at $\Delta t / 2$, where the pressure term is at $t$ instant, according to Eq. (1).

$$
\widetilde{U}_{i}^{n+1 / 2}=U_{i}^{n}-\frac{\Delta t}{2}\left(\frac{\partial f_{i j}^{n}}{\partial x_{j}}-\frac{\partial \tau_{i j}^{n}}{\partial x_{j}}+\frac{\partial p^{n}}{\partial x_{i}}-\rho g_{i}-w_{j}^{n} \frac{\partial U_{i}^{n}}{\partial x_{i}}\right)(1)
$$

where $\rho$ is the density, $p$ is the pressure, $g_{i}$ are the gravity acceleration components, $v_{i}$ are the velocity components, $\mathrm{w}_{i}$ are the velocity components of the reference system and $\tau_{i j}$ is the viscous stress tensor $U_{i}=\rho_{\mathrm{V}_{i}}, f_{i j}=\mathrm{v}_{j}\left(\rho_{\mathrm{V}_{i}}\right)=\mathrm{v}_{j} U_{i}(i, j=1,2,3)$.

(b) Update the pressure $p$ at $t+\Delta t$, given by the Poisson equation:

$$
\frac{1}{c^{2}} \Delta p=-\Delta t\left[\frac{\partial \widetilde{U}_{i}^{n+1 / 2}}{\partial x_{i}}-\frac{\Delta t}{4} \frac{\partial}{\partial x_{i}} \frac{\partial \Delta p}{\partial x_{i}}\right]
$$

where $\Delta p=p^{n+1}-p^{n}$ and $i=1,2,3$.

(c) Correct the velocity at $t+\Delta t / 2$, adding the pressure variation term from $t$ to $t+\Delta t / 2$, according to the equation:

$$
U_{i}^{n+1 / 2}=\widetilde{U}_{i}^{n+1 / 2}-\frac{\Delta t}{4} \frac{\partial \Delta p}{\partial x_{i}}
$$

(d) Calculate the velocity at $t+\Delta t$ using variables updated in the previous steps as follows:

$U_{i}^{n+1}=U_{i}^{n}$

$-\Delta t\left(\frac{\partial f_{i j}^{n+1 / 2}}{\partial x_{j}}-\frac{\partial \tau_{i j}^{n+1 / 2}}{\partial x_{j}}+\frac{\partial p^{n+1 / 2}}{\partial x_{i}}-w_{j}^{n+1 / 2} \frac{\partial U_{i}^{n+1 / 2}}{\partial x_{i}}-\rho g_{i}\right)(4)$

\section{Space discretization}

The classical Galerkin weighted residual method is applied to the space discretization. In the variables at $t+\Delta t / 2$ instant, a constant shape function $\mathbf{P E}$ is used, and in the variables at $t$ and $t+\Delta t$, a linear shape function $\mathbf{N}$ is employed. By applying this procedure to Eq. (1), (2), (3) and (4), the following expressions in the matrix form are obtained (Teixeira and Awruch, 2001):

$$
\begin{aligned}
& \Omega_{E}^{n+1 / 2} \widetilde{\mathbf{U}}_{i}^{n+1 / 2}=\mathbf{C} \overline{\mathbf{U}}_{i}^{n} \\
& -\frac{\Delta t}{2}\left[\mathbf{L}_{j}\left(\overline{\mathbf{f}}_{i j}^{n}-\overline{\boldsymbol{\tau}}_{i j}^{n}\right)+\mathbf{L}_{i} \overline{\mathbf{p}}^{n}-\mathbf{T} \overline{\mathbf{U}}_{i}^{n}-\Omega_{E}^{n+1 / 2} \overline{\rho g} \bar{g}_{i}\right] \\
& \left(\tilde{\mathbf{M}}+\frac{\Delta t^{2}}{4} \mathbf{H}\right) \Delta \overline{\mathbf{p}}=\Delta t\left(\mathbf{L}_{i}^{T} \widetilde{\overline{\mathbf{U}}}_{i}^{n+1 / 2}+\mathbf{f}_{a}\right) \\
& \overline{\mathbf{U}}_{i}^{n+1 / 2}={\widetilde{\mathbf{U}_{i}}}^{n+1 / 2}-\frac{\Delta t}{4 \Omega_{E}} \mathbf{L}_{i} \Delta \overline{\mathbf{p}} \\
& \mathbf{M}^{n+1} \overline{\mathbf{U}}_{i}^{n+1}=\mathbf{M}^{n} \overline{\mathbf{U}}_{i}^{n}+\Delta t \mathbf{L}_{j}^{T}\left(\overline{\mathbf{f}}_{i j}^{n+1 / 2}-\overline{\mathbf{W}}_{j}^{n+1 / 2} \overline{\mathbf{U}}_{i}^{n+1 / 2}\right) \\
& -\Delta t \mathbf{Q}_{j} \bar{\tau}_{i j}^{n}+\Delta t \mathbf{Q}_{i}\left(\overline{\mathbf{p}}^{n}+\Delta \overline{\mathbf{p}} / 2\right)+\Delta t \mathbf{S}_{b i}-\Delta t \mathbf{C}^{T} \overline{\mathbf{g}}_{i}
\end{aligned}
$$

where variables with upper bars at $n$ and $n+1$ instants indicate nodal values, while those at $n+1 / 2$ instant represent constant values in the element. The matrices and vectors from Eq. (5) to (8) are volume and surface integrals that can be seen in detail in Teixeira and Awruch (2000).

Equation (6) is solved using the conjugated gradient method with diagonal pre-conditioning (Argyris et al., 1985). In Eq. (8), the consistent mass matrix is substituted by the lumped mass matrix, and then this equation is solved iteratively.

The scheme is conditionally stable and the local stability condition for the element $E$ is given by

$$
\Delta t_{E} \leq \beta h_{E} /|\mathbf{u}|
$$

where $h_{\mathrm{E}}$ is the characteristic element size, $\beta$ is the safety factor and $\mathbf{u}$ is the fluid velocity.

\section{Mesh movement}

The free surface is the interface between two fluids, water and air, where atmospheric pressure is considered constant (generally the reference value is null). In this interface, the kinematic free surface boundary condition (KFSBC) is imposed. By using the ALE formulation, it is expressed as:

$$
\frac{\partial \eta}{\partial t}+{ }^{(s)} \mathrm{v}_{i} \frac{\partial \eta}{\partial x_{i}}={ }^{(s)} \mathrm{v}_{3}
$$

where $\eta$ is the free surface elevation, ${ }^{(s)} \mathrm{v}_{3}$ is the vertical fluid velocity component and ${ }^{(s)} \mathrm{v}_{i} \quad(i=1,2)$ are the horizontal fluid velocity components in the free surface. The eulerian formulation is used in the $x_{1}$ and $x_{2}$ directions (horizontal plane) while the ALE formulation is employed in the $x_{3}$ or vertical direction.

The time discretization of KFSBC is carried out in the same way as the one for the momentum equations as presented before. After applying 
expansion in Taylor series, the expressions for $\eta$ at $n+1 / 2$ (first step) and $n+1$ (second step) instants are obtained:

$$
\begin{aligned}
& \eta^{n+1 / 2}=\eta^{n}+\frac{\Delta t}{2}\left({ }^{(s)} \mathrm{v}_{3}-{ }^{(s)} \mathrm{v}_{1} \frac{\partial \eta}{\partial x_{1}}-{ }^{(s)} \mathrm{v}_{2} \frac{\partial \eta}{\partial x_{2}}\right)^{n} \\
& \eta^{n+1}=\eta^{n}+\Delta t\left({ }^{(s)} \mathrm{v}_{3}-{ }^{(s)} \mathrm{v}_{1} \frac{\partial \eta}{\partial x_{1}}-{ }^{(s)} \mathrm{v}_{2} \frac{\partial \eta}{\partial x_{2}}\right)^{n+1 / 2}
\end{aligned}
$$

Linear triangular elements coincident with the face of the tetrahedral elements on the free surface are used to the space discretization by applying the Galerkin method.

The mesh velocity vertical component $\mathrm{w}_{3}$ is computed to diminish element distortions, keeping prescribed velocities on moving (free surface) and stationary (bottom) boundary surfaces. The mesh movement algorithm adopted in this paper uses a smoothing procedure for the velocities based on these boundary surfaces. The updating of the mesh velocity at point $i$ of the finite element domain is based on the mesh velocity of the points $j$ that belong to the boundary surfaces, and is expressed in the following way (Teixeira and Awruch, 2005):

$$
w_{3}^{i}=\frac{\sum_{j=1}^{n s} a_{i j} w_{3}^{j}}{\sum_{j=1}^{n s} a_{i j}}
$$

where $n s$ is the total number of points belonging to the boundary surfaces and $a_{i j}$ are the influence coefficients between the point $i$ inside the domain and the point $j$ on the boundary surface given by the following expression:

$$
a_{i j}=\frac{1}{d_{i j}^{4}}
$$

with $d_{i j}$ being the distance between points $i$ and $j$. In other words, $a_{i j}$ represents the weight that every point $j$ on the boundary surface has on the value of the mesh velocity at points $i$ inside the domain. When $d_{i j}$ is low, $a_{i j}$ has a high value, favouring the influence of points $i$, located closer to the boundary surface containing point $j$.

The free surface elevation, the mesh velocity and the vertical coordinate are updated according to the following steps:

(1) Calculate $\eta^{n+1 / 2}$ and $\widetilde{U}_{i}^{n+1 / 2}$, Eq. (11) and

Eq. (1), respectively.

(2) Calculate $\Delta p$, Eq. (2).

(3) Calculate $U_{i}^{n+1 / 2}$, Eq. (3).

(4) Calculate $U_{i}^{n+1}$, Eq. (4).

(5) Calculate $\eta^{n+1}$, Eq. (12)

(6) Update the mesh velocity $\mathrm{w}_{3}$ and the vertical coordinate $x_{3}$ :
(6.1) Calculate the mesh velocity in the free surface at $t+\Delta t:{ }^{(S)} \mathbf{w}_{3}^{n+1}=\left(\eta^{n+1}-\eta^{n}\right) / \Delta t$.

(6.2) Calculate the mesh velocity in the interior of the domain at $n+1$ e $n+1 / 2$ by using Eq. (13) and $\mathrm{w}_{3}^{n+1 / 2}=\frac{\left(\mathrm{w}_{3}^{n+1}+\mathrm{w}_{3}^{n}\right)}{2}$, respectively.

(6.3) Update the vertical coordinates in the interior of the domain: $x_{3}^{n+1 / 2}=x_{3}^{n}+\mathrm{w}_{3}^{n} \frac{\Delta t}{2}$, $x_{3}^{n+1}=x_{3}^{n}+w_{3}^{n+1 / 2} \Delta \mathrm{t}$.

\section{Wave generation and radiation conditions}

The free surface elevation and the fluid velocity components are imposed to each time step directly, considering the linear wave equations.

The Flather's radiation condition (Flather, 1976) is used to deal with open boundaries. In this method, the Sommerfeld condition to free surface elevation is combined with one-dimensional version of the continuity equation. Then, the normal velocity of the boundary can be expressed by:

$$
u=\eta \sqrt{\frac{g}{h}}
$$

where $g$ is the gravity acceleration and $h$ is the depth.

\section{STUDY CASE}

This case considers a $5.2 \mathrm{~m}$ long and $0.425 \mathrm{~m}$ deep channel with a submerged cylinder of $\mathrm{r}=0.025 \mathrm{~m}$ positioned $1.60 \mathrm{~m}$ from the wave generator (Fig. 1). The cylinder center is $0.075 \mathrm{~m} \mathrm{(3r)}$ from the free surface. The frequency wave is $f=1.4 \mathrm{~Hz}$; its amplitude is $a=0.0119 \mathrm{~m}$ and its wavelength is $L=0.796 \mathrm{~m}$, characterizing a deep water case.

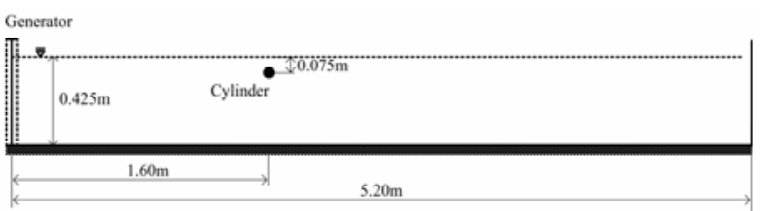

Figure 1. Geometry of the horizontal cylinder case.

Table 1 shows the period, the frequency and the wavelength for the fundamental frequency and its 2nd, 3rd and 4th harmonics, according to the linear wave theory.

Table 1. Period, frequency and wavelength of the fundamental frequency and its $2 \mathrm{nd}, 3 \mathrm{rd}$ and 4 th harmonics.

\begin{tabular}{|c|c|c|c|c|}
\hline & Fundamental & $\begin{array}{c}\mathbf{2}^{\text {nd }} \\
\text { harmonic }\end{array}$ & $\begin{array}{c}\mathbf{3}^{\text {rd }} \\
\text { harmonic }\end{array}$ & $\begin{array}{c}\mathbf{4}^{\text {th }} \\
\text { harmonic }\end{array}$ \\
\hline $\boldsymbol{T}(\mathbf{s})$ & 0.7143 & 0.3571 & 0.2381 & 0.1786 \\
\hline $\boldsymbol{f ( H z )}$ & 1.4 & 2.8 & 4.2 & 5.6 \\
\hline $\boldsymbol{L}(\mathbf{m})$ & 0.796 & 0.199 & 0.0885 & 0.0498 \\
\hline
\end{tabular}


The mesh, with 173900 nodes and 515623 elements, has one layer of elements in the transversal direction. The average element size on the cylinder boundary is $0.0015 \mathrm{~m} \mathrm{(105} \mathrm{divisions} \mathrm{in} \mathrm{the}$ circumference). The element size diminishes from the ends to the region near the cylinder and from the bottom to the free surface. The element sizes on the end where the wave generator is located and on the

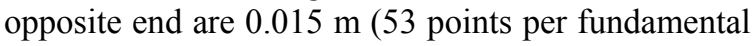
wavelength) and $0.02 \mathrm{~m}$ (40 points per fundamental wavelength), respectively. On the bottom, $0.0015 \mathrm{~m}$ is also used.

The initial conditions are: null velocity components in all domain and hydrostatic pressure (null on the free surface). The wave is generated by imposing the surface elevation and the velocity components. The non-slip condition is imposed to the bottom and to the cylinder wall. The time step is $0.0002 \mathrm{~s}$, which satisfies the Courant condition.

\section{RESULTS AND DISCUSSIONS}

Figure 2 shows the free surface elevation obtained by the code and experimental tests, where $x C$ is the horizontal coordinate of the cylinder center. In general, there is agreement between numerical and experimental results (Paixão Conde, 2007). We can notice the free surface disturbance downstream the cylinder. When $(x-x c) / L$ is above 1.7 , the numerical results are smoother than the experimental ones, showing the necessity of a refinement in this region.

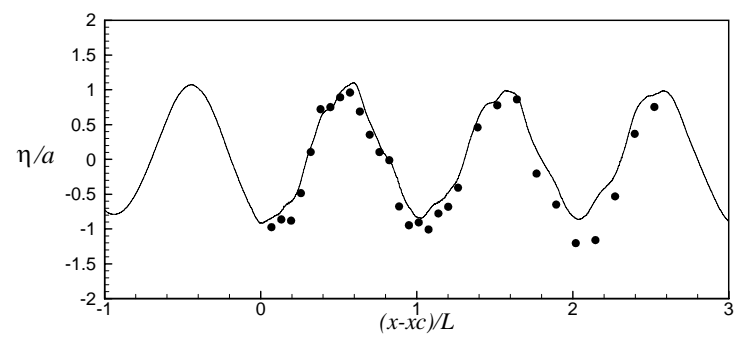

Figure 2. Free surface elevation ( Numerical - ; Experimental $\mathbf{a})$.

Figure 3 shows a comparison among numerical and experimental results in terms of free surface elevation on four gauges located at $(x-x c) / L$ equal to $-0.503,0.0692,0.509$ and 1.264 (there is only a numerical result on the first gauge). We can observe the similarity among numerical and experimental results.

Figure 4 shows the streamlines and the velocity modulus distribution at the same instant used in Fig. 2. Recirculation and separation cannot be observed at downstream. Due to the oscillatory flow behavior, there is no time for recirculation productions. We can notice the flow acceleration near the cylinder due to the boundary layer effect. The viscous effects have only local influence, without modifying the velocity field far from it.
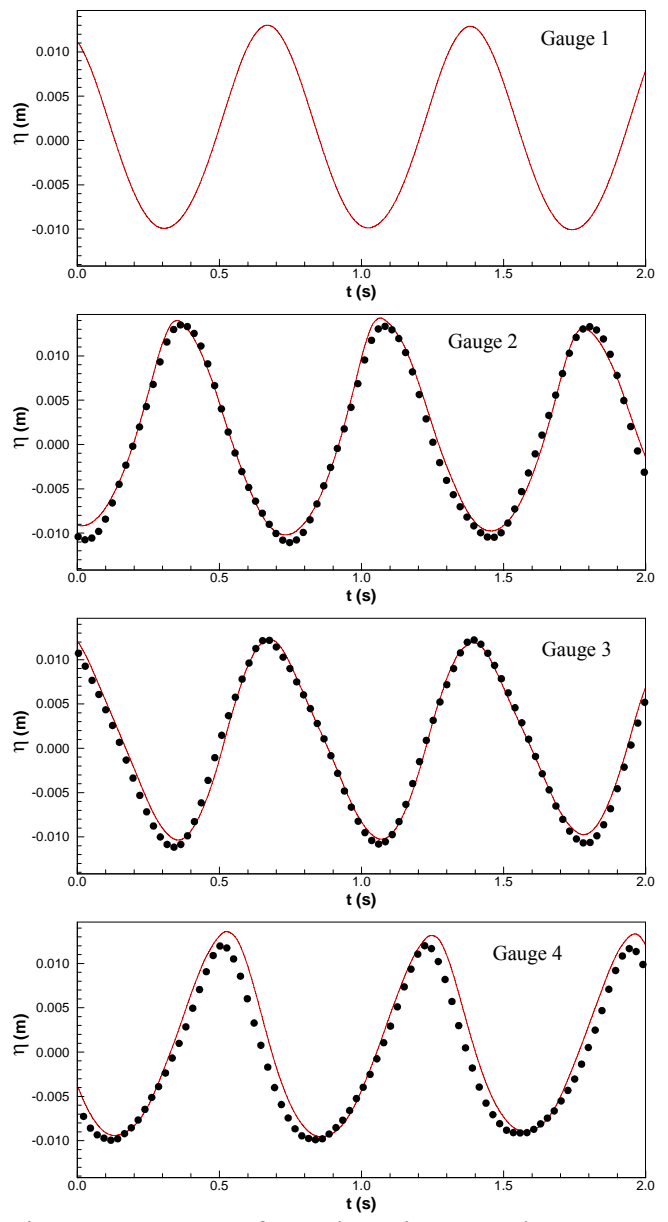

Figure 3. Free surface elevations on the gauges located at $(x-X c) / L$ equal to $-0.503,0.0692,0.509$ and 1.264 (Numerical — ; Experimental •).

In Figures 5 and 6, velocity component profiles, $\mathrm{u}$ and $\mathrm{v}$, on the same gauge positions are presented. These profiles were constructed at the same instant as that used in Fig. 2. According to the linear theory, the maximum value for both horizontal and vertical components is equal to $0.105 \mathrm{~m} / \mathrm{s}$. For horizontal components, these values occur on the crest and the trough, while for vertical ones, these values occur on upward and downward zero-crossings. When one component is the maximum, another is null, because the phase difference is 90 degrees.

Gauge $1((x-X c) / L=-0.503)$ is located upstream, near the wave crest; no significant disturbance in $\mathrm{u}$ and $\mathrm{v}$ profiles is observed. The horizontal velocity component is positive and its maximum value is similar to the theoretical value in the crest. The wave trough passes by gauge 2 ; the vertical velocity component presents low values and the horizontal velocity component has negative values, reaching the maximum absolute value close to the theoretical ones $(0.105 \mathrm{~m} / \mathrm{s})$. Gauge 3 is located near the first crest upstream, resulting in high horizontal component values. Finally, gauge 4 is on a region between the trough and upward zero-crossing. Both component profiles are negative and the vertical component 
magnitude shows how close the gauge is to upward zero-crossing.

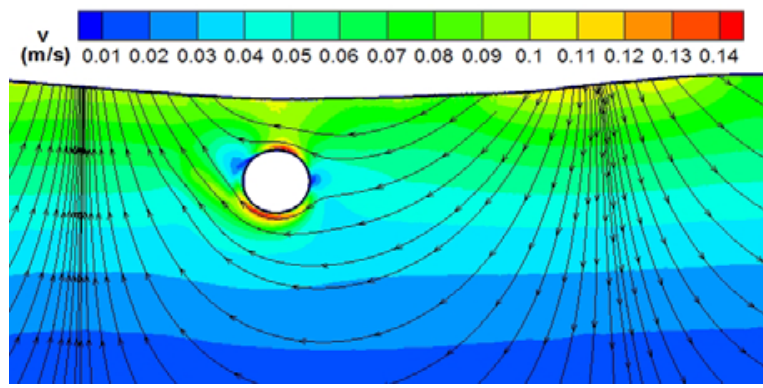

Figure 4. Streamlines and velocity modulus at the instant in which the free-surface elevation was captured (Fig. 2).

The non-slip boundary condition on the bottom does not change the general behavior of the wave propagation, because this case is considered a deep water one.
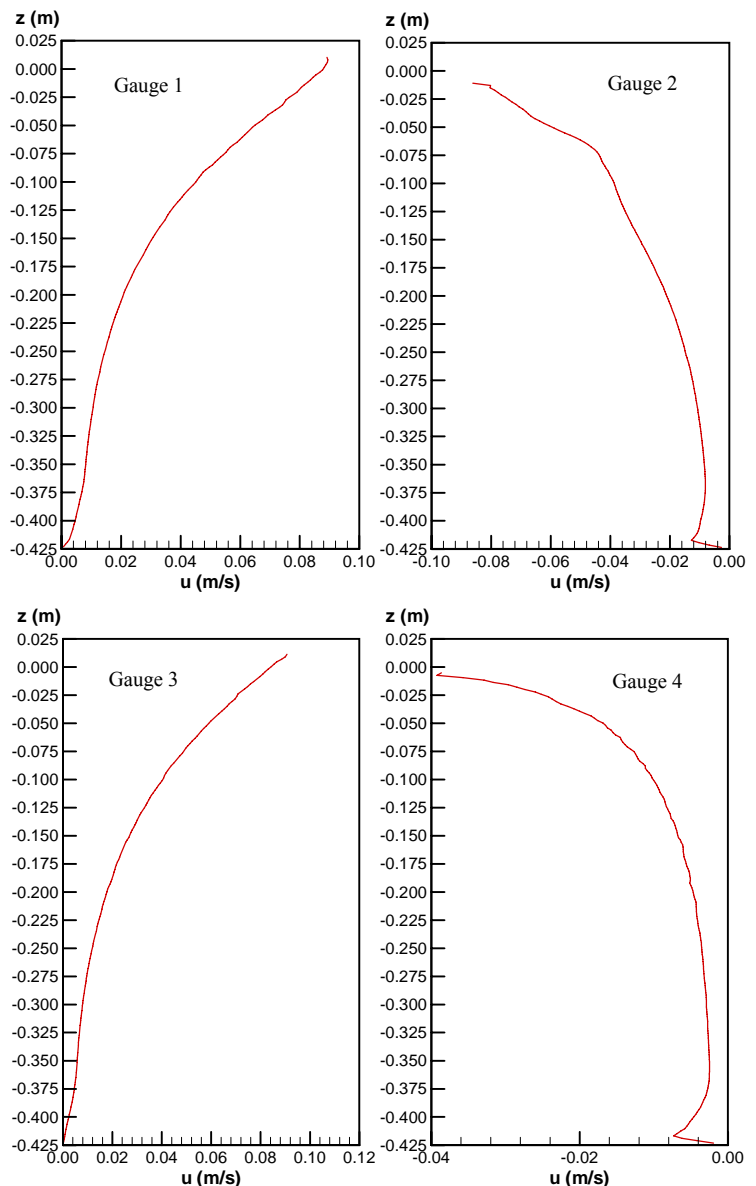

Figure 5. Horizontal velocity components at the same instant used in Fig. 2 along the depth on gauges located at $(x-X c) / L$ equal to $-0.503,0.0692,0.509$ and 1.264 .
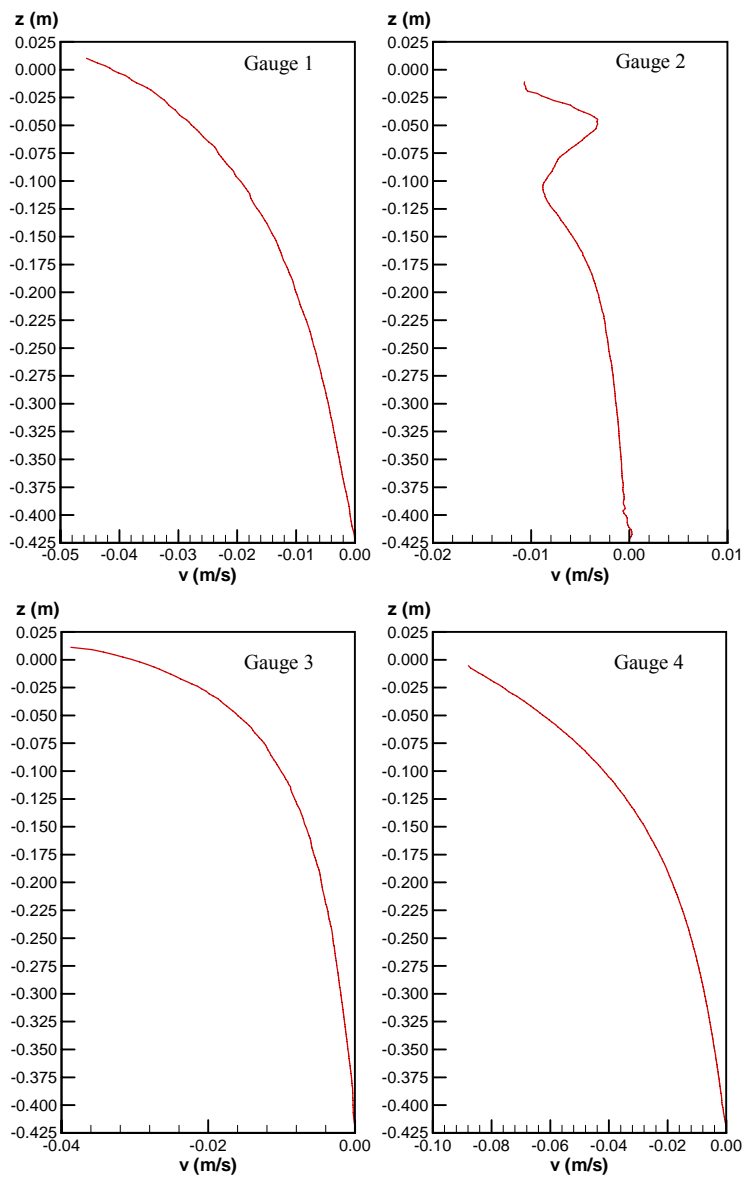

Figure 6. Vertical velocity components at the same instant used in Fig. 2 along the depth on gauges located at $(x-x c) / L$ equal to $-0.503,0.0692,0.509$ and 1.264 .

Figure 7 shows the frequency spectra for these four gauges distributed along the channel. In all cases, the energy is concentrated on the fundamental frequency and its harmonic waves. On gauge 1, the fundamental frequency presents most energy and the second harmonic shows a little value. On gauges 2 and 4, located upstream, significant energy up to the third harmonic appears, similar to the experimental results.
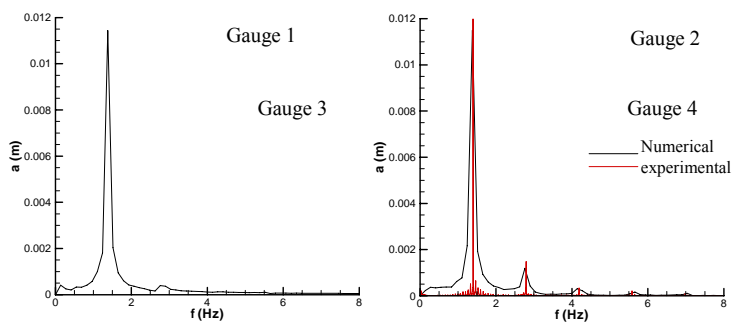


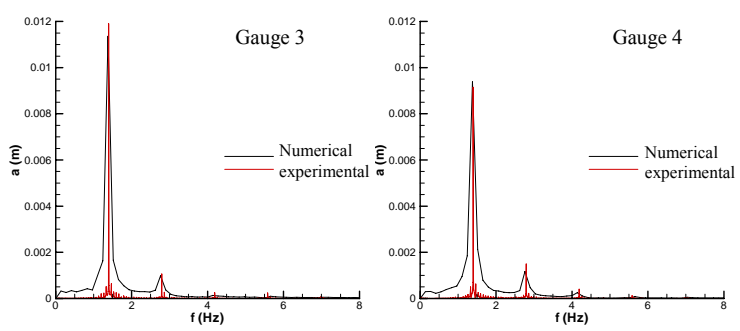

Figure 7. Frequency spectra on gauges located at $(x-x c) / L$ equal to $-0.503,0.0692,0.509$ e 1.264 .

\section{CONCLUSIONS}

This paper has presented a validation of a code based on the fractioned semi-implicit two-step Taylor-Galerkin method to wave propagation along a channel with submerged obstacle. A horizontal cylinder case was studied and the numerical results were compared with experimental ones.

The free surface elevations and the velocity profiles obtained by the code were similar to experimental ones. The numerical results presented a smooth free surface deformation downstream, possibly because of the lack of refinement that caused numerical diffusion. In this case, the viscous effects influenced the flow behavior locally whereas the viscosity was not important far from the cylinder. The non-slip condition on the bottom did not modify the wave propagation significantly because it is a deep water case.

\section{ACKNOWLEDGEMENT}

The author greatly acknowledges Miguel Lopes (that provided the experimental data), Eric Didier and J. M. Paixão Conde and the partnership between FURG and LNEC.

\section{REFERENCES}

Argyris, J., St. Doltsinis, J., Wuestenberg, H., and Pimenta, P. M., 1985, Finite element solution of viscous flow problems, Finite Elements in Fluids.Wiley, New York, Vol.6, pp. 89-114.

Chaplin, J. R., 2001, Nonlinear Wave Interactions with a Submerged Horizontal Cylinder, Proc. 11th Int. Offshore and Polar Eng. Conf., Stavanger, Vol. 3, pp. 272-279.

Dean, W. R., 1948, On the Reflection of Surface Waves by a Submerged Circular Cylinder, Proc. Camb. Phil. Soc., Vol. 44, pp. 483-491.

Flather, R. A., 1976, A tidal model of the northwest European continental shelf, Mem. Soc. R. Liege, Ser., Vol. 10, No. 6, pp. 141-164.

Paixão Conde, J. M., Didier, E., Lopes, M. F. P., and Gato, L.M.C., 2007, Nonlinear wave diffraction by a submerged horizontal circular cylinder, Proc. 17th Int. Offshore and Polar Eng. Conf. (ISOPE 2007), Lisbon.

Schonberg, T., and Chaplin, J. R., 2003, Computation of Nonlinear Wave Reflections and Transmissions from Submerged Horizontal Cylinder, Int. J. Offshore and Polar Eng., Vol.13, pp. 29-37.

Teixeira, P. R. F., 2001, Simulação numérica da interação de escoamentos tridimensionais de fluidos compressíveis e incompressíveis e estruturas deformáveis usando o método de elementos finitos, Doctoral thesis, PPGEC-UFRGS, Porto Alegre, RS. (in Portuguese).

Teixeira, P. R. F., and Awruch, A. M., 2000, Numerical simulation of three dimensional incompressible flows using the finite element method, 8th Brazilian Congress of Thermal Engineering and Sciences - ENCIT, Porto Alegre.

Teixeira, P. R. F., and Awruch, A. M., 2005, Numerical simulation of fluid-structure interaction using the finite element method, Computers \& Fluids, Vol. 34, pp. 249-273.

Ursell, F., 1950, Surface Waves on Deep Water in presence of a Submerged Circular Cylinder, Proc. Camb. Phil. Soc., Vol. 46, pp. 141-158.

Received: September 04, 2008

Revised: October 04, 2008

Accepted: November 04, 2008 